\title{
The LAMBADA dataset: Word prediction requiring a broad discourse context*
}

\author{
Denis Paperno, Germán Kruszewski, Angeliki Lazaridou, Quan Ngoc Pham ${ }^{\dagger}$, \\ Raffaella Bernardi, Sandro Pezzelle, Marco Baroni, Gemma Boleda, Raquel Fernández ${ }^{\ddagger}$ \\ CIMeC - Center for Mind/Brain Sciences, University of Trento \\ \{firstname.lastname\}@unitn.it, ${ }^{\dagger}$ quanpn90@gmail.com \\ ${ }_{\ddagger}^{\ddagger}$ Institute for Logic, Language \& Computation, University of Amsterdam \\ raquel. fernandez@uva.nl
}

\begin{abstract}
We introduce LAMBADA, a dataset to evaluate the capabilities of computational models for text understanding by means of a word prediction task. LAMBADA is a collection of narrative passages sharing the characteristic that human subjects are able to guess their last word if they are exposed to the whole passage, but not if they only see the last sentence preceding the target word. To succeed on LAMBADA, computational models cannot simply rely on local context, but must be able to keep track of information in the broader discourse. We show that LAMBADA exemplifies a wide range of linguistic phenomena, and that none of several state-ofthe-art language models reaches accuracy above $1 \%$ on this novel benchmark. We thus propose LAMBADA as a challenging test set, meant to encourage the development of new models capable of genuine understanding of broad context in natural language text.
\end{abstract}

\section{Introduction}

The recent spurt of powerful end-to-end-trained neural networks for Natural Language Processing (Hermann et al., 2015; Rocktäschel et al., 2016; Weston et al., 2015, a.o.) has sparked interest in tasks to measure the progress they are bringing about in genuine language understanding. Special care must be taken in evaluating such systems, since their effectiveness at picking statistical generalizations from large corpora can lead to the illusion that they are reaching a deeper degree of understanding than they really are. For example,

\footnotetext{
${ }^{*}$ Denis and Germán share first authorship. Marco, Gemma, and Raquel share senior authorship.
}

the end-to-end system of Vinyals and Le (2015), trained on large conversational datasets, produces dialogues such as the following:

$$
\begin{aligned}
& \text { Human: what is your job? } \\
& \text { Machine: i'm a lawyer } \\
& \text { Human: what do you do? } \\
& \text { Machine: i'm a doctor }
\end{aligned}
$$

Separately, the system responses are appropriate for the respective questions. However, when taken together, they are incoherent. The system behaviour is somewhat parrot-like. It can locally produce perfectly sensible language fragments, but it fails to take the meaning of the broader discourse context into account. Much research effort has consequently focused on designing systems able to keep information from the broader context into memory, and possibly even perform simple forms of reasoning about it (Hermann et al., 2015; Hochreiter and Schmidhuber, 1997; Ji et al., 2015; Mikolov et al., 2015; Sordoni et al., 2015; Sukhbaatar et al., 2015; Wang and Cho, 2015, a.o.).

In this paper, we introduce the LAMBADA dataset (LAnguage Modeling Broadened to Account for Discourse Aspects). LAMBADA proposes a word prediction task where the target item is difficult to guess (for English speakers) when only the sentence in which it appears is available, but becomes easy when a broader context is presented. Consider Example (1) in Figure 1. The sentence Do you honestly think that I would want you to have a _-_. ? has a multitude of possible continuations, but the broad context clearly indicates that the missing word is miscarriage.

LAMBADA casts language understanding in the classic word prediction framework of language modeling. We can thus use it to test several existing language modeling architectures, including 
systems with capacity to hold longer-term contextual memories. In our preliminary experiments, none of these models came even remotely close to human performance, confirming that LAMBADA is a challenging benchmark for research on automated models of natural language understanding.

\section{Related datasets}

The CNN/Daily Mail (CNNDM) benchmark recently introduced by Hermann et al. (2015) is closely related to LAMBADA. CNNDM includes a large set of online articles that are published together with short summaries of their main points. The task is to guess a named entity that has been removed from one such summary. Although the data are not normed by subjects, it is unlikely that the missing named entity can be guessed from the short summary alone, and thus, like in LAMBADA, models need to look at the broader context (the article). Differences between the two datasets include text genres (news vs. novels; see Section 3.1) and the fact that missing items in $\mathrm{CN}$ NDM are limited to named entities. Most importantly, the two datasets require models to perform different kinds of inferences over broader passages. For CNNDM, models must be able to summarize the articles, in order to make sense of the sentence containing the missing word, whereas in LAMBADA the last sentence is not a summary of the broader passage, but a continuation of the same story. Thus, in order to succeed, models must instead understand what is a plausible development of a narrative fragment or a dialogue.

Another related benchmark, CBT, has been introduced by Hill et al. (2016). Like LAMBADA, CBT is a collection of book excerpts, with one word randomly removed from the last sentence in a sequence of 21 sentences. While there are other design differences, the crucial distinction between CBT and LAMBADA is that the CBT passages were not filtered to be human-guessable in the broader context only. Indeed, according to the post-hoc analysis of a sample of CBT passages reported by Hill and colleagues, in a large proportion of cases in which annotators could guess the missing word from the broader context, they could also guess it from the last sentence alone. At the same time, in about one fifth of the cases, the annotators could not guess the word even when the broader context was given. Thus, only a small portion of the CBT passages are really probing the model's ability to understand the broader context, which is instead the focus of LAMBADA.

The idea of a book excerpt completion task was originally introduced in the MSRCC dataset (Zweig and Burges, 2011). However, the latter limited context to single sentences, not attempting to measure broader passage understanding.

Of course, text understanding can be tested through other tasks, including entailment detection (Bowman et al., 2015), answering questions about a text (Richardson et al., 2013; Weston et al., 2015) and measuring inter-clause coherence (Yin and Schütze, 2015). While different tasks can provide complementary insights into the models' abilities, we find word prediction particularly attractive because of its naturalness (it's easy to norm the data with non-expert humans) and simplicity. Models just need to be trained to predict the most likely word given the previous context, following the classic language modeling paradigm, which is a much simpler setup than the one required, say, to determine whether two sentences entail each other. Moreover, models can have access to virtually unlimited amounts of training data, as all that is required to train a language model is raw text. On a more general methodological level, word prediction has the potential to probe almost any aspect of text understanding, including but not limited to traditional narrower tasks such as entailment, co-reference resolution or word sense disambiguation.

\section{The LAMBADA dataset}

\subsection{Data collection ${ }^{1}$}

LAMBADA consists of passages composed of a context (on average 4.6 sentences) and a target sentence. The context size is the minimum number of complete sentences before the target sentence such that they cumulatively contain at least 50 tokens (this size was chosen in a pilot study). The task is to guess the last word of the target sentence (the target word). The constraint that the target word be the last word of the sentence, while not necessary for our research goal, makes the task more natural for human subjects.

The LAMBADA data come from the Book Corpus (Zhu et al., 2015). The fact that it contains unpublished novels minimizes the potential

\footnotetext{
${ }^{1}$ Further technical details are provided in the Supplementary Material (SM): http://clic.cimec.unitn.it/ lambada /
} 
(1) Context: "Yes, I thought I was going to lose the baby." "I was scared too," he stated, sincerity flooding his eyes. "You were ?" "Yes, of course. Why do you even ask?" "This baby wasn't exactly planned for."

Target sentence: "Do you honestly think that I would want you to have a ?"

Target word: miscarriage

(2) Context: "Why?" "I would have thought you'd find him rather dry," she said. "I don't know about that," said Gabriel. "He was a great craftsman," said Heather. "That he was," said Flannery.

Target sentence: "And Polish, to boot," said

Target word: Gabriel

(3) Context: Preston had been the last person to wear those chains, and I knew what I'd see and feel if they were slipped onto my skin-the Reaper's unending hatred of me. I'd felt enough of that emotion already in the amphitheater. I didn't want to feel anymore. "Don't put those on me," I whispered. "Please."

Target sentence: Sergei looked at me, surprised by my low, raspy please, but he put down the

Target word: chains

(4) Context: They tuned, discussed for a moment, then struck up a lively jig. Everyone joined in, turning the courtyard into an even more chaotic scene, people now dancing in circles, swinging and spinning in circles, everyone making up their own dance steps. I felt my feet tapping, my body wanting to move.

Target sentence: Aside from writing, I 've always loved

Target word: dancing

(5) Context: He shook his head, took a step back and held his hands up as he tried to smile without losing a cigarette. "Yes you can," Julia said in a reassuring voice. "I 've already focused on my friend. You just have to click the shutter, on top, here."

Target sentence: He nodded sheepishly, through his cigarette away and took the

Target word: camera

(6) Context: In my palm is a clear stone, and inside it is a small ivory statuette. A guardian angel. "Figured if you're going to be out at night getting hit by cars, you might as well have some backup." I look at him, feeling stunned. Like this is some sort of sign.

Target sentence: But as I stare at Harlin, his mouth curved in a confident grin, I don't care about

Target word: signs

(7) Context: Both its sun-speckled shade and the cool grass beneath were a welcome respite after the stifling kitchen, and I was glad to relax against the tree's rough, brittle bark and begin my breakfast of buttery, toasted bread and fresh fruit. Even the water was tasty, it was so clean and cold.

Target sentence: It almost made up for the lack of

Target word: coffee

(8) Context: My wife refused to allow me to come to Hong Kong when the plague was at its height and -" "Your wife, Johanne? You are married at last ?" Johanne grinned. "Well, when a man gets to my age, he starts to need a few home comforts.

Target sentence: After my dear mother passed away ten years ago now, I became

Target word: lonely

(9) Context: "Again, he left that up to you. However, he was adamant in his desire that it remain a private ceremony. He asked me to make sure, for instance, that no information be given to the newspaper regarding his death, not even an obituary.

Target sentence: I got the sense that he didn't want anyone, aside from the three of us, to know that he'd even

Target word: died

(10) Context: The battery on Logan's radio must have been on the way out. So he told himself. There was no other explanation beyond Cygan and the staff at the White House having been overrun. Lizzie opened her eyes with a flutter. They had been on the icy road for an hour without incident.

Target sentence: Jack was happy to do all of the

Target word: driving

Figure 1: Examples of LAMBADA passages. Underlined words highlight when the target word (or its lemma) occurs in the context. 
usefulness of general world knowledge and external resources for the task, in contrast to other kinds of texts like news data, Wikipedia text, or famous novels. The corpus, after duplicate removal and filtering out of potentially offensive material with a stop word list, contains 5,325 novels and 465 million words. We randomly divided the novels into equally-sized training and development+testing partitions. We built the LAMBADA dataset from the latter, with the idea that models tackling LAMBADA should be trained on raw text from the training partition, composed of 2662 novels and encompassing more than 200M words. Because novels are pre-assigned to one of the two partitions only, LAMBADA passages are self-contained and cannot be solved by exploiting the knowledge in the remainder of the novels, for example background information about the characters involved or the properties of the fictional world in a given novel. The same novel-based division method is used to further split LAMBADA data between development and testing.

To reduce time and cost of dataset collection, we filtered out passages that are relatively easy for standard language models, since such cases are likely to be guessable based on local context alone. We used a combination of four language models, chosen by availability and/or ease of training: a pre-trained recurrent neural network (RNN) (Mikolov et al., 2011) and three models trained on the Book Corpus (a standard 4-gram model, a RNN and a feed-forward model; see SM for details, and note that these are different from the models we evaluated on LAMBADA as described in Section 4 below). Any passage whose target word had probability $\geq 0.00175$ according to any of the language models was excluded.

A random sample of the remaining passages were then evaluated by human subjects through the CrowdFlower crowdsourcing service ${ }^{2}$ in three steps. For a given passage,

1. one human subject guessed the target word based on the whole passage (comprising the context and the target sentence); if the guess was right,

2. a second subject guessed the target word based on the whole passage; if that guess was also right,

3. more subjects tried to guess the target word based on the target sentence only, until the

\footnotetext{
${ }^{2}$ http: //www. crowdflower.com
}

word was guessed or the number of unsuccessful guesses reached 10; if no subject was able to guess the target word, the passage was added to the LAMBADA dataset.

The subjects in step 3 were allowed 3 guesses per sentence, to maximize the chances of catching cases where the target words were guessable from the sentence alone. Step 2 was added based on a pilot study that revealed that, while step 3 was enough to ensure that the data could not be guessed with the local context only, step 1 alone did not ensure that the data were easy given the discourse context (its output includes a mix of cases ranging from obvious to relatively difficult, guessed by an especially able or lucky step-1 subject). We made sure that it was not possible for the same subject to judge the same item in both passage and sentence conditions (details in SM).

In the crowdsourcing pipeline, $84-86 \%$ items were discarded at step 1, an additional 6-7\% at step 2 and another 3-5\% at step 3. Only about one in 25 input examples passed all the selection steps.

Subjects were paid $\$ 0.22$ per page in steps 1 and 2 (with 10 passages per page) and $\$ 0.15$ per page in step 3 (with 20 sentences per page). Overall, each item in the resulting dataset costed \$1.24 on average. Alternative designs, such as having step 3 before step 2 or before step 1, were found to be more expensive. Cost considerations also precluded us from using more subjects at stage 1, which could in principle improve the quality of filtering at this step.

Note that the criteria for passage inclusion were very strict: We required two consecutive subjects to exactly match the missing word, and we made sure that no subject (out of ten) was able to provide it based on local context only, even when given 3 guesses. An alternative to this perfect-match approach would have been to include passages where broad-context subjects provided other plausible or synonymous continuations. However, it is very challenging, both practically and methodologically, to determine which answers other than the original fit the passage well, especially when the goal is to distinguish between items that are solvable in broad-discourse context and those where the local context is enough. Theoretically, substitutability in context could be tested with manual annotation by multiple additional raters, but this would not be financially or practically feasible for a dataset of this scale (human annotators received 
over 200,000 passages at stage 1). For this reason we went for the strict hit-or-miss approach, keeping only items that can be unambiguously determined by human subjects.

\subsection{Dataset statistics}

The LAMBADA dataset consists of 10,022 passages, divided into 4,869 development and 5,153 test passages (extracted from 1,331 and 1,332 disjoint novels, respectively). The average passage consists of 4.6 sentences in the context plus 1 target sentence, for a total length of 75.4 tokens (dev) / 75 tokens (test). Examples of passages in the dataset are given in Figure 1.

The training data for language models to be tested on LAMBADA include the full text of 2,662 novels (disjoint from those in dev+test), comprising 203 million words. Note that the training data consists of text from the same domain as the dev+test passages, in large amounts but not filtered in the same way. This is partially motivated by economic considerations (recall that each data point costs $\$ 1.24$ on average), but, more importantly, it is justified by the intended use of LAMBADA as a tool to evaluate general-purpose models in terms of how they fare on broad-context understanding (just like our subjects could predict the missing words using their more general text understanding abilities), not as a resource to develop ad-hoc models only meant to predict the final word in the sort of passages encountered in LAMBADA. The development data can be used to fine-tune models to the specifics of the LAMBADA passages.

\subsection{Dataset analysis}

Our analysis of the LAMBADA data suggests that, in order for the target word to be predictable in a broad context only, it must be strongly cued in the broader discourse. Indeed, it is typical for LAMBADA items that the target word (or its lemma) occurs in the context. Figure 2(a) compares the LAMBADA items to a random 5000-item sample from the input data, that is, the passages that were presented to human subjects in the filtering phase (we sampled from all passages passing the automated filters described in Section 3.1 above, including those that made it to LAMBADA). The figure shows that when subjects guessed the word (only) in the broad context, often the word itself occurred in the context: More than $80 \%$ of LAMBADA passages include the target word in the context, while in the input data that was the case for less than $15 \%$ of the passages. To guess the right word, however, subjects must still put their linguistic and general cognitive skills to good use, as shown by the examples featuring the target word in the context reported in Figure 1.

Figure 2(b) shows that most target words in LAMBADA are proper nouns (48\%), followed by common nouns (37\%) and, at a distance, verbs $(7.7 \%)$. In fact, proper nouns are hugely overrepresented in LAMBADA, while the other categories are under-represented, compared to the POS distribution in the input. A variety of factors converges in making proper nouns easy for subjects in the LAMBADA task. In particular, when the context clearly demands a referential expression, the constraint that the blank be filled by a single word excludes other possibilities such as noun phrases with articles, and there are reasons to suspect that co-reference is easier than other discourse phenomena in our task (see below). However, although co-reference seems to play a big role, only $0.3 \%$ of target words are pronouns.

Common nouns are still pretty frequent in LAMBADA, constituting over one third of the data. Qualitative analysis reveals a mixture of phenomena. Co-reference is again quite common (see Example (3) in Figure 1), sometimes as "partial" co-reference facilitated by bridging mechanisms (shutter-camera; Example (5)) or through the presence of a near synonym ('lose the baby'miscarriage; Example (1)). However, we also often find other phenomena, such as the inference of prototypical participants in an event. For instance, if the passage describes someone having breakfast together with typical food and beverages (see Example (7)), subjects can guess the target word coffee without it having been explicitly mentioned.

In contrast, verbs, adjectives, and adverbs are rare in LAMBADA. Many of those items can be guessed with local sentence context only, as shown in Figure 2(b), which also reports the POS distribution of the set of items that were guessed by subjects based on the target-sentence context only (step 3 in Section 3.1). Note a higher proportion of verbs, adjectives and adverbs in the latter set in Figure 2(b). While end-of-sentence context skews input distribution in favour of nouns, subject filtering does show a clear differential effect for nouns vs. other POSs. Manual inspection reveals that broad context is not necessary to guess items like 


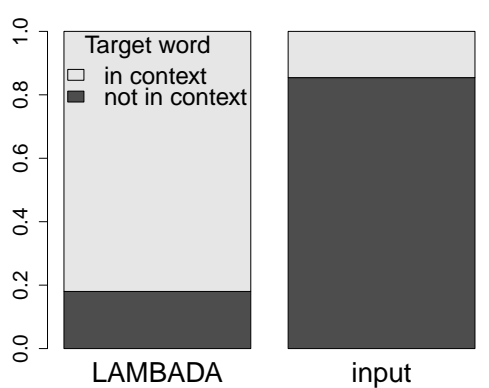

(a)

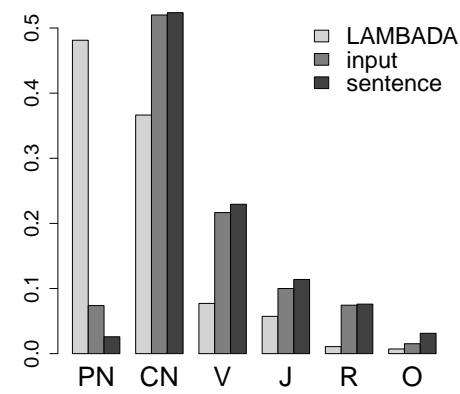

(b)

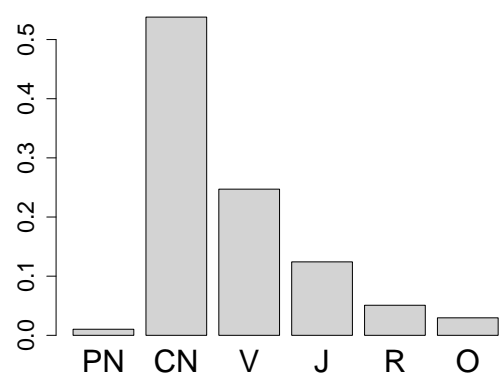

(c)

Figure 2: (a) Target word in or not in context; (b) Target word POS distribution in LAMBADA vs. data presented to human subjects (input) and items guessed with sentence context only (PN=proper noun, $\mathrm{CN}=$ common noun, $\mathrm{V}=\mathrm{verb}, \mathrm{J}=$ adjective, $\mathrm{R}=\mathrm{adverb}, \mathrm{O}=\mathrm{other}$ ); (c) Target word POS distribution of LAMBADA passages where the lemma of the target word is not in the context (categories as in (b)).

frequent verbs (ask, answer, call), adjectives, and closed-class adverbs (now, too, well), as well as time-related adverbs (quickly, recently). In these cases, the sentence context suffices, so few of them end up in LAMBADA (although of course there are exceptions, such as Example (8), where the target word is an adjective). This contrasts with other types of open-class adverbs (e.g., innocently, confidently), which are generally hard to guess with both local and broad context. The low proportion of these kinds of adverbs and of verbs among guessed items in general suggests that tracking event-related phenomena (such as script-like sequences of events) is harder for subjects than coreferential phenomena, at least as framed in the LAMBADA task. Further research is needed to probe this hypothesis.

Furthermore, we observe that, while explicit mention in the preceding discourse context is critical for proper nouns, the other categories can often be guessed without having been explicitly introduced. This is shown in Figure 2(c), which depicts the POS distribution of LAMBADA items for which the lemma of the target word is not in the context (corresponding to about $16 \%$ of LAMBADA in total). ${ }^{3}$ Qualitative analysis of items with verbs and adjectives as targets suggests that the target word, although not present in the passage, is still strongly implied by the con-

\footnotetext{
${ }^{3}$ The apparent $1 \%$ of out-of-context proper nouns shown in Figure 2(c) is due to lemmatization mistakes (fictional characters for which the lemmatizer did not recognize a link between singular and plural forms, e.g., Wynn - Wynns). A manual check confirmed that all proper noun target words in LAMBADA are indeed also present in the context.
}

text. In about one third of the cases examined, the missing word is "almost there". For instance, the passage contains a word with the same root but a different part of speech (e.g., death-died in Example (6)), or a synonymous expression (as mentioned above for "miscarriage"; we find the same phenomenon for verbs, e.g., 'deprived you of water'-dehydrated).

In other cases, correct prediction requires more complex discourse inference, including guessing prototypical participants of a scene (as in the coffee example above), actions or events strongly suggested by the discourse (see Examples (1) and (10), where the mention of an icy road helps in predicting the target driving), or qualitative properties of participants or situations (see Example (8)). Of course, the same kind of discourse reasoning takes place when the target word is already present in the context (cf. Examples (3) and (4)). The presence of the word in context does not make the reasoning unnecessary (the task remains challenging), but facilitates the inference.

As a final observation, intriguingly, the LAMBADA items contain (quoted) direct speech significantly more often than the input items overall (71\% of LAMBADA items vs. $61 \%$ of items in the input sample), see, e.g., Examples (1) and (2). Further analysis is needed to investigate in what way more dialogic discourse might facilitate the prediction of the final target word.

In sum, LAMBADA contains a myriad of phenomena that, besides making it challenging from the text understanding perspective, are of great interest to the broad Computational Linguistics 
community. To return to Example (1), solving it requires a combination of linguistic skills ranging from (morpho)phonology (the plausible target word abortion is ruled out by the indefinite determiner $a$ ) through morphosyntax (the slot should be filled by a common singular noun) to pragmatics (understanding what the male participant is inferring from the female participant's words), in addition to general reasoning skills. It is not surprising, thus, that LAMBADA is so challenging for current models, as we show next.

\section{Modeling experiments}

Computational methods We tested several existing language models and baselines on LAMBADA. We implemented a simple RNN (Elman, 1990), a Long Short-Term Memory network (LSTM) (Hochreiter and Schmidhuber, 1997), a traditional statistical N-Gram language model (Stolcke, 2002) with and without cache, and a Memory Network (Sukhbaatar et al., 2015). We remark that at least LSTM, Memory Network and, to a certain extent, the cache N-Gram model have, among their supposed benefits, the ability to take broader contexts into account. Note moreover that variants of RNNs and LSTMs are at the state of the art when tested on standard language modeling benchmarks (Mikolov, 2014). Our Memory Network implementation is similar to the one with which Hill et al. (2016) reached the best results on the CBT data set (see Section 2 above). While we could not re-implement the models that performed best on CNNDM (see again Section 2), our LSTM is architecturally similar to the Deep LSTM Reader of Hermann et al. (2015), which achieved respectable performance on that data set. Most importantly, we will show below that most of our models reach impressive performance when tested on a more standard language modeling data set sourced from the same corpus used to build LAMBADA. This control set was constructed by randomly sampling $5 \mathrm{~K}$ passages of the same shape and size as the ones used to build LAMBADA from the same test novels, but without filtering them in any way. Based on the control set results, to be discussed below, we can reasonably claim that the models we are testing on LAMBADA are very good at standard language modeling, and their low performance on the latter cannot be attributed to poor quality.

In order to test for strong biases in the data, we constructed Sup-CBOW, a baseline model weakly tailored to the task at hand, consisting of a simple neural network that takes as input a bag-ofword representation of the passage and attempts to predict the final word. The input representation comes from adding pre-trained $\mathrm{CBOW}$ vectors (Mikolov et al., 2013) of the words in the passage. ${ }^{4}$ We also considered an unsupervised variant (Unsup-CBOW) where the target word is predicted by cosine similarity between the passage vector and the target word vector. Finally, we evaluated several variations of a random guessing baseline differing in terms of the word pool to sample from. The guessed word could be picked from: the full vocabulary, the words that appear in the current passage and a random uppercased word from the passage. The latter baseline aims at exploiting the potential bias that proper names account for a consistent portion of the LAMBADA data (see Figure 2 above).

Note that LAMBADA was designed to challenge language models with harder-than-average examples where broad context understanding is crucial. However, the average case should not be disregarded either, since we want language models to be able to handle both cases. For this reason, we trained the models entirely on unsupervised data and expect future work to follow similar principles. Concretely, we trained the models, as is standard practice, on predicting each upcoming word given the previous context, using the LAMBADA training data (see Section 3.2 above) as input corpus. The only exception to this procedure was Sup-CBOW where we extracted from the training novels similar-shaped passages to those in LAMBADA and trained the model on them (about $9 \mathrm{M}$ passages). Again, the goal of this model was only to test for potential biases in the data and not to provide a full account for the phenomena we are testing. We restricted the vocabulary of the models to the $60 \mathrm{~K}$ most frequent words in the training set (covering $95 \%$ of the target words in the development set). The model hyperparameters were tuned on their accuracy in the development set. The same trained models were tested on the LAMBADA and the control sets. See SM for the tuning details.

Results Results of models and baselines are reported in Table 1. Note that the measure of interest

\footnotetext{
${ }^{4}$ http://clic.cimec.unitn.it/composes / semantic-vectors.html
} 


\begin{tabular}{|l|l|c|c|c|}
\hline Data & Method & Acc. & Ppl. & Rank \\
\hline \multirow{5}{*}{ LAMBADA } & \multicolumn{3}{|c|}{ baselines } \\
\cline { 2 - 5 } & Random vocabulary word & 0 & 60000 & 30026 \\
& Random word from passage & 1.6 & - & - \\
& Random capitalized word from passage & 7.3 & - & - \\
& Unsup-CBOW & 0 & 57040 & 16352 \\
& Sup-CBOW & 0 & 47587 & 4660 \\
\cline { 2 - 5 } & models \\
\cline { 2 - 5 } & N-Gram & 0.1 & 3125 & 993 \\
& N-Gram w/cache & 0.1 & 768 & 87 \\
& RNN & 0 & 14725 & 7831 \\
& LSTM & 0 & 5357 & 324 \\
& Memory Network & 0 & 16318 & 846 \\
\hline \multirow{5}{*}{ baselines } \\
\cline { 2 - 5 } & Random vocabulary word & 0 & 60000 & 30453 \\
& Random word from passage & 0 & - & - \\
& Random capitalized word from passage & 0 & - & - \\
& Unsup-CBOW & 0 & 55190 & 12950 \\
& Sup-CBOW & 3.5 & 2344 & 259 \\
\cline { 2 - 6 } & \multicolumn{3}{|c|}{} \\
\cline { 2 - 5 } & N-Gram models & 19.1 & 285 & 17 \\
& N-Gram w/cache & 19.1 & 270 & 18 \\
& RNN & 15.4 & 277 & 24 \\
& LSTM & 21.9 & 149 & 12 \\
& Memory Network & 566 & 46 \\
\hline
\end{tabular}

Table 1: Results of computational methods. Accuracy is expressed in percentage.

for LAMBADA is the average success of a model at predicting the target word, i.e., accuracy (unlike in standard language modeling, we know that the missing LAMBADA words can be precisely predicted by humans, so good models should be able to accomplish the same feat, rather than just assigning a high probability to them). However, as we observe a bottoming effect with accuracy, we also report perplexity and median rank of correct word, to better compare the models.

As anticipated above, and in line with what we expected, all our models have very good performance when called to perform a standard language modeling task on the control set. Indeed, 3 of the models (the N-Gram models and LSTM) can guess the right word in about $1 / 5$ of the cases.

The situation drastically changes if we look at the LAMBADA results, where all models are performing very badly. Indeed, no model is even able to compete with the simple heuristics of picking a random word from the passage, and, especially, a random capitalized word (easily a proper noun). At the same time, the low performance of the latter heuristic in absolute terms (7\% accuracy) shows that, despite the bias in favour of names in the passage, simply relying on this will not suffice to obtain good performance on LAMBADA, and models should rather pursue deeper forms of analysis of the broader context (the Sup-CBOW baseline, attempting to directly exploit the passage in a shallow way, performs very poorly). This confirms again that the difficulty of LAMBADA relies mainly on accounting for the information available in a broader context and not on the task of predicting the exact word missing.

In comparative terms (and focusing on perplexity and rank, given the uniformly low accuracy results) we observe a stronger performance of the traditional N-Gram models over the neuralnetwork-based ones, possibly pointing to the difficulty of tuning the latter properly. In particular, the best relative performance on LAMBADA is achieved by N-Gram w/cache, which takes passage statistics into account. While even this model is effectively unable to guess the right word, it achieves a respectable perplexity of 768 . 
We recognize, of course, that the evaluation we performed is very preliminary, and it must only be taken as a proof-of-concept study of the difficulty of LAMBADA. Better results might be obtained simply by performing more extensive tuning, by adding more sophisticated mechanisms such as attention (Bahdanau et al., 2014), and so forth. Still, we would be surprised if minor modifications of the models we tested led to human-level performance on the task.

We also note that, because of the way we have constructed LAMBADA, standard language models are bound to fail on it by design: one of our first filters (see Section 3.1) was to choose passages where a number of simple language models were failing to predict the upcoming word. However, future research should find ways around this inherent difficulty. After all, humans were still able to solve this task, so a model that claims to have good language understanding ability should be able to succeed on it as well.

\section{Conclusion}

This paper introduced the new LAMBADA dataset, aimed at testing language models on their ability to take a broad discourse context into account when predicting a word. A number of linguistic phenomena make the target words in LAMBADA easy to guess by human subjects when they can look at the whole passages they come from, but nearly impossible if only the last sentence is considered. Our preliminary experiments suggest that even some cutting-edge neural network approaches that are in principle able to track long-distance effects are far from passing the LAMBADA challenge.

We hope the computational community will be stimulated to develop novel language models that are genuinely capturing the non-local phenomena that LAMBADA reflects. To promote research in this direction, we plan to announce a public competition based on the LAMBADA data. ${ }^{5}$

Our own hunch is that, despite the initially disappointing results of the "vanilla" Memory Network we tested, the ability to store information in a longer-term memory will be a crucial component of successful models, coupled with the ability to perform some kind of reasoning about what's

\footnotetext{
${ }^{5}$ The development set of LAMBADA, along with the training corpus, can be downloaded at http://clic. cimec.unitn.it/lambada/. The test set will be made available at the time of the competition.
}

stored in memory, in order to retrieve the right information from it.

On a more general note, we believe that leveraging human performance on word prediction is a very promising strategy to construct benchmarks for computational models that are supposed to capture various aspects of human text understanding. The influence of broad context as explored by LAMBADA is only one example of this idea.

\section{Acknowledgments}

We are grateful to Aurelie Herbelot, Tal Linzen, Nghia The Pham and, especially, Roberto Zamparelli for ideas and feedback. This project has received funding from the European Union's Horizon 2020 research and innovation programme under the Marie Sklodowska-Curie grant agreement No 655577 (LOVe); ERC 2011 Starting Independent Research Grant n. 283554 (COMPOSES); NWO VIDI grant n. 276-89-008 (Asymmetry in Conversation). We gratefully acknowledge the support of NVIDIA Corporation with the donation of the GPUs used in our research.

\section{References}

Dzmitry Bahdanau, Kyunghyun Cho, and Yoshua Bengio. 2014. Neural machine translation by jointly learning to align and translate. In ICLR.

Samuel Bowman, Gabor Angeli, Christopher Potts, and Christopher Manning. 2015. A large annotated corpus for learning natural language inference. In Proceedings of EMNLP, pages 632-642, Lisbon, Portugal.

Jeffrey L Elman. 1990. Finding structure in time. Cognitive science, 14(2):179-211.

Karl Moritz Hermann, Tomáš Kočiský, Edward Grefenstette, Lasse Espeholt, Will Kay, Mustafa Suleyman, and Phil Blunsom. 2015. Teaching machines to read and comprehend. In Proceedings of NIPS, Montreal, Canada. Published online: https://papers.nips.cc/book/ advances-in-neural-informationprocessing-systems-28-2015.

Felix Hill, Antoine Bordes, Sumit Chopra, and Jason Weston. 2016. The Goldilocks principle: Reading children's books with explicit memory representations. In Proceedings of ICLR Conference Track, San Juan, Puerto Rico. Published online: http://www.iclr.cc/doku.php?id= iclr2016:main.

Sepp Hochreiter and Jürgen Schmidhuber. 1997. Long short-term memory. Neural Computation, 9(8):1735-178-. 
Yangfeng Ji, Trevor Cohn, Lingpeng Kong, Chris Dyer, and Jacob Eisenstein. 2015. Document context language models. http://arxiv.org/abs/ 1511.03962 .

Tomas Mikolov, Stefan Kombrink, Anoop Deoras, Lukar Burget, and Jan Honza Cernocky. 2011. Rnnlm - recurrent neural network language. In Proceedings of ASRU. IEEE Automatic Speech Recognition and Understanding Workshop.

Tomas Mikolov, Kai Chen, Greg Corrado, and Jeffrey Dean. 2013. Efficient estimation of word representations in vector space. http://arxiv.org/ $\mathrm{abs} / 1301.3781 /$.

Tomas Mikolov, Armand Joulin, Sumit Chopra, Michael Mathieu, and Marc' Aurelio Ranzato. 2015. Learning longer memory in recurrent neural networks. In Proceedings of ICLR Workshop Track, San Diego, CA. Published online: http://www. iclr.cc/doku.php?id=iclr2015:main.

Tomas Mikolov. 2014. Using neural networks for modelling and representing natural languages. Slides presented at COLING, online at http://www.coling2014.org/COLING \2014\Tutorialfix $\backslash-\backslash$ TomasMikolov.pdf.

Matthew Richardson, Christopher Burges, and Erin Renshaw. 2013. MCTest: A challenge dataset for the open-domain machine comprehension of text. In Proceedings of EMNLP, pages 193-203, Seattle, WA.

Tim Rocktäschel, Edward Grefenstette, Karl Moritz Hermann, Tomáš Kočiský, and Phil Blunsom. 2016. Reasoning about entailment with neural attention. In Proceedings of ICLR Conference Track, San Juan, Puerto Rico. Published online: http://www. iclr.cc/doku.php?id=iclr2016:main.

Alessandro Sordoni, Michel Galley, Michael Auli, Chris Brockett, Yangfeng Ji, Margaret Mitchell, Jian-Yun Nie, Jianfeng Gao, and Bill Dolan. 2015. A neural network approach to context-sensitive generation of conversational responses. In Proceedings of NAACL, pages 196-205, Denver, CO.

Andreas Stolcke. 2002. Srilm-an extensible language modeling toolkit. In INTERSPEECH, volume 2002, page 2002 .

Sainbayar Sukhbaatar, Arthur Szlam, Jason Weston, and Rob Fergus. 2015. End-to-end memory networks. http://arxiv.org/abs/1503. 08895.

Oriol Vinyals and Quoc Le. 2015. A neural conversational model. In Proceedings of the ICML Deep Learning Workshop, Lille, France. Published online: https://sites.google.com/site/ deeplearning2015/accepted-papers.
Tian Wang and Kyunghyun Cho. 2015. Largercontext language modelling. http://arxiv. org/abs/1511.03729.

Jason Weston, Antoine Bordes, Sumit Chopra, and Tomas Mikolov. 2015. Towards AI-complete question answering: A set of prerequisite toy tasks. http: //arxiv.org/abs/1502.05698.

Wenpeng Yin and Hinrich Schütze. 2015. MultiGranCNN: An architecture for general matching of text chunks on multiple levels of granularity. In Proceedings of ACL, pages 63-73, Beijing, China.

Yukun Zhu, Ryan Kiros, Richard Zemel, Ruslan Salakhutdinov, Raquel Urtasun, Antonio Torralba, and Sanja Fidler. 2015. Aligning books and movies: Towards story-like visual explanations by watching movies and reading books. In ICCV 2015, pages 19-27.

Geoffrey Zweig and Christopher Burges. 2011. The Microsoft Research sentence completion challenge. Technical Report MSR-TR-2011-129, Microsoft Research. 